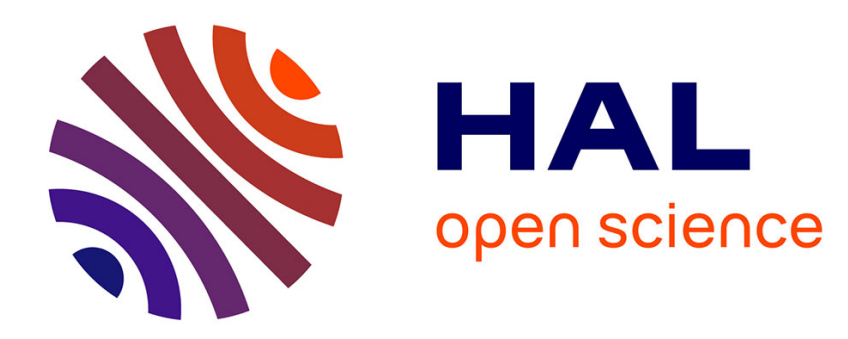

\title{
Oltre il fallimento. La prima Jugoslavia, una storia europea
}

Fabio Giomi, Stefano Petrungaro

\section{To cite this version:}

Fabio Giomi, Stefano Petrungaro. Oltre il fallimento. La prima Jugoslavia, una storia europea. Passato e Presente, 2020, 110, pp.107-121. 10.3280/PASS2020-110007 . hal-03094630

\section{HAL Id: hal-03094630 \\ https://hal.science/hal-03094630}

Submitted on 4 Jan 2021

HAL is a multi-disciplinary open access archive for the deposit and dissemination of scientific research documents, whether they are published or not. The documents may come from teaching and research institutions in France or abroad, or from public or private research centers.
L'archive ouverte pluridisciplinaire HAL, est destinée au dépôt et à la diffusion de documents scientifiques de niveau recherche, publiés ou non, émanant des établissements d'enseignement et de recherche français ou étrangers, des laboratoires publics ou privés. 


\title{
Oltre il fallimento. La prima Jugoslavia, una storia europea
}

\author{
Fabio Giomi*, Stefano Petrungaro**
}

Beyond Failure. The first Yugoslavia, a European history

According to an established scholarship, the history of interwar Yugoslavia can be mainly interpreted through the lens of the national question and authoritarianism. This contribution aims at challenging this view, stressing how this country was also the stage of innovative forms of governance, shaped by complex interactions between state and non-state actors. What emerges is an original Yugoslav response to the crisis of European liberalism.

Keywords: Yugoslavia, corporatism, religious institutions, voluntary associations, interwar Europe

Parole-chiave: Jugoslavia, corporativismo, istituzioni religiose, associazioni volontarie, Europe tra le due guerre mondiali

\section{Per una rilettura della storia jugoslava tra le due guerre mondiali}

La Jugoslavia tra le due guerre mondiali ha attratto l'attenzione degli studiosi meno rispetto ai periodi precedenti e successivi. Gli studi sulla prima Jugoslavia - fondata nel 1918 come Regno dei Serbi, Croati e Sloveni, poi Regno di Jugoslavia nel 1929 - sono caratterizzati da alcuni capisaldi: quella jugoslava sarebbe una società irrimediabilmente spaccata lungo linee etniche predeterminate; la sua storia sarebbe sostanzialmente interpretabile attraverso il prisma della questione nazionale; il suo sistema economico e politico si caratterizzerebbe per arretratezza, inefficienza e brutalità contro i gruppi etnici minoritari. Secondo queste letture, non sorprende che dopo un primo decennio di parlamentarismo balbuziente, il paese scivoli naturalmente verso un sistema autoritario e repressivo, con l'introduzione della dittatura reale nel 1929 e il rapido collasso dopo l'invasione delle truppe dell'Asse nel 1941. Prendendo in prestito una categoria del giornalismo, saremmo di fronte a un caso di failed state, condannato in partenza da fratture insolubili, sorta di prologo delle

* CNRS, Centre d'études turques, ottomanes, balkaniques et centrasiatiques, 54 boulevard Raspail, 75006 Paris, France; fabio.giomi@ehess.fr

** Università Ca' Foscari, Dorsoduro 1405, Fondamenta Zattere, Palazzo Cosulich, 30123 Venezia; stefano.petrungaro@unive.it 
terribili guerre di dissoluzione jugoslave che avrebbero chiuso questo violento XX secolo europeo ${ }^{1}$.

La prima Jugoslavia è stata analizzata dagli storici soprattutto attraverso il prisma della storia politica tradizionale, concentrata sui partiti, l'élite parlamentare e governativa, gli intellettuali contigui ad essi. Un tale approccio non ha lasciato molto spazio a personalità e istituzioni ai margini dell'apparato statale o che non facessero parte di esso, ossia gli attori della cosiddetta società civile. Approcci simili hanno l'indubbio merito di aver scandagliato a fondo il tema dei conflitti nazionali e della repressione politica, questioni che qui non verranno richiamate perché già ampiamente discusse negli studi esistenti. Quella prospettiva analitica, però, presenta il forte rischio di ridurre l'intensa storia di quei decenni a una vicenda di inevitabili conflitti e violenze, lasciando in ombra altre dinamiche altrettanto rilevanti per la comprensione di quella fase storica.

Sulla scorta di studi recenti, siamo infatti convinti che quella della prima Jugoslavia non sia una storia governata dall'ineluttabilità, e che l'insieme dei princìpi e delle procedure che hanno regolato le relazioni fra stato e società in questa parte dell'Europa tra le due guerre mondiali non debbano essere liquidate semplicemente come inadeguate e brutali. Diverse opere pubblicate negli ultimi anni suggeriscono di ripensare la prima Jugoslavia come un contesto nel quale vari attori - pubblici e privati, civili e religiosi - hanno cercato soluzioni, mai stabili, mai codificate in un'unica sintesi ideologica, per la costruzione di una comunità politica vivibile. La tesi che vogliamo proporre è che la Jugoslavia interbellica, e probabilmente l'intera Europa del Sud-Est ${ }^{2}$, è un laboratorio di pratiche politiche originali, prodotto in parte delle eredità statali ottomana e asburgica, in parte della circolazione transnazionale di persone, idee e beni, agevolata dai progressi tecnologici degli anni '20 e '30. Intendendo lo stato come ente che non solo impone norme e istituzioni a una società recalcitrante, parliamo di governance, concetto mutuato dalla scienza politica per «abbattere il privilegio politico dello Stato», concependo il sistema politico come un complesso «sistema di interazioni e interdipendenze» tra attori sociali di natura diversa ${ }^{3}$. Utilizziamo questa controversa categoria analitica per sottolineare l'importanza di concentrarsi sulla «cooperazione e l'interazione tra

\footnotetext{
${ }^{1}$ I. Banac, The National Question in Yugoslavia. Origins, History, Politics, Cornell UP, Ithaca 1984; S.P. Ramet, The Three Yugoslavias. State-Building and Legitimation, 1918-2005, Indiana UP, Washington 2006; J. Pirjevec, Il giorno di San Vito. Jugoslavia 1918-1992, storia di una tragedia, Nuova ERI, Torino 1993; H. Matković, Povijest Jugoslavije, Naklada Pavičić, Zagreb 1998.

${ }^{2} \mathrm{Cfr}$. in italiano sul Sud-est Europa A. Basciani, L'illusione della modernità. Il sudest dell'Europa tra le due guerre mondiali, Rubbettino, Soveria Mannelli 2016; E. Costantini, Nae Ionescu, Mircea Eliade, Emil Cioran. Antiliberalismo nazionalista alla periferia d'Europa, Morlacchi, Perugia 2005; F. Guida (a cura di), Intellettuali versus democrazia. I regimi autoritari in Europa sud-orientale (1933-1953), Carocci, Roma 2010.

${ }^{3}$ J. Crowley, Usages de la gouvernance et de la gouvernementalité, «Critique internationale», 21 (2003), n. 4, pp. 52-61.
} 
lo stato e attori non-statuali all'interno di reti decisionali miste pubblico/private» ${ }^{4}$. Le relazioni fra stato e società nel periodo fra le due guerre mondiali non si possono leggere solo attraverso la coppia interpretativa repressione dall'alto/resistenza dal basso; sono invece il risultato, continuamente testato e contestato, di interazioni più complesse tra progetti e aspirazioni provenienti da diversi settori dello stato e della società.

Un'altra prospettiva che ci sembra promettente è quella di uscire dall'ottica centro-periferia, che a lungo ha dominato gli studi storici sull'Europa del SudEst, in base alla quale questa regione sarebbe un angolo del continente marginale e passivo, dominato da legami prepolitici basati sul sangue e la religione, mero ricettacolo di discorsi e pratiche politiche elaborate altrove. Gli studi sul «balcanismo» hanno invece sottolineato i caratteri transnazionali delle tradizioni politiche balcaniche, che si inseriscono-in contesti più ampi ${ }^{5}$. Anche le forme di governance elaborate tra le due guerre in Jugoslavia possono essere lette alla luce del contesto europeo e del Mediterraneo orientale. La prima Jugoslavia appare così una variazione originale sul tema della ricerca di soluzioni alla crisi del liberalismo europeo ${ }^{6}$.

Per illuminare questo complesso scenario, abbiamo individuato tre temi: la rappresentanza degli interessi collettivi in chiave corporativa, la sfera dell'associazionismo e infine quella delle istituzioni religiose. Questi tre approfondimenti permettono di osservare alcuni casi rilevanti di quell'ambigua quanto intrigante interazione tra attori privati, religiosi e professionali da un lato, e pubblici, statali, regionali e municipali, dall'altro.

\section{Esplorazioni corporativiste}

Un ambito che potrebbe gettare una nuova luce sulle trasformazioni della statualità in Jugoslavia e nel Sud-est Europa tra le due guerre è quello delle nuove forme di mediazione tra Stato e società di natura corporativa ${ }^{7}$. ¿̀ un

\footnotetext{
${ }^{4}$ Cfr. R. Mayntz, La teoria della governance: sfide e prospettive, «Rivista italiana di scienze politiche», 29 (1999), n. 1, pp. 3-21.

${ }^{5}$ A partire dal pionieristico saggio di M. Todorova, Immaginando i Balcani (1997), Argo, Lecce 2002, cfr. Ead., The Trap of Backwardness: Modernity, Temporality, and the Study of Eastern European Nationalism, «Slavic Review», 64 (2005), 1, pp. 14064; A. Anastassiadis-N. Clayer (ed.), Society, Politics and State-Formation in SouthEastern Europe during the 19th century, Alpha Bank Historical Archives, Athens 2011; S. Rutar (ed.), Beyond the Balkans: Towards an Inclusive History of Southeastern Europe, Lit Verlag, Münster 2014; Ead., L'Europa sudorientale e la storia comparata europea, «Passato e presente», 81 (2010), pp. 105-120.

${ }^{6}$ Ch.S. Maier, La rifondazione dell'Europa borghese (1975), il Mulino, Bologna 1999; E.J. Hobsbawm, Il secolo breve (1994), Rizzoli, Milano 1995, pp. 135-71; M. Mazower, Le ombre dell'Europa. Democrazie e totalitarismi nel XX secolo (1998), Garzanti, Milano 2005.

${ }^{7}$ Per uno sguardo comparativo: G. Santomassimo, La terza via fascista. Il mito del corporativismo, Carocci, Roma 2006; M. Pasetti, L'Europa corporativa. Una storia transnazionale tra le due guerre mondiali, Bononia UP, Bologna 2016; A. Costa Pinto (ed.), Corporatism and Fascism. The Corporatist Wave in Europe, Palgrave, London 2017.
} 
aspetto relativamente trascurato dagli studi, sebbene sia generalmente riconosciuto che elementi di stampo corporativista caratterizzassero le teorie politiche di numerosi attori sociali e politici ${ }^{8}$.

Ideali d'origine corporativa erano assai diffusi, ad esempio, tra gli intellettuali cattolici croati. Un eminente rappresentate di un corporativismo di matrice cristiano-sociale è Juraj Šćetinec, docente di sociologia ed esperto di politiche sociali. Grande estimatore dell'enciclica Quadragesimo Anno di Pio $\mathrm{XI}$, seguiva attentamente gli sviluppi socio-politici in tutta l'Europa centrale, oltre che in Austria, Germania e in Italia. Scettico nei confronti del fascismo italiano, riteneva che il corporativismo fosse inconciliabile con la limitazione della libertà individuale o dei gruppi professionali, suddividendo il campo corporativista idealmente in due gruppi, quello fascista e nazional-socialista, detti «universali-totalitari», e un democratico «corporativismo cristiano», dall'altro'.

La tradizione cristiano-sociale era assai diffusa anche in Slovenia, dove il partito dominante era il Partito Popolare Sloveno (Slovenska ljudska stranka) guidato dal prete cattolico Anton Korošec. In Slovenia la tradizione clericale era assai radicata e la Chiesa cattolica era la principale interprete di ideali corporativi, che negli anni ' 30 confluirono nel programma socio-economico del movimento cattolico sloveno. Per il nuovo comune stato jugoslavo si prevedeva un'organizzazione strutturata attraverso sei camere provinciali, nelle quali avrebbero dovuto sedere rappresentanti di ogni organizzazione socioeconomica. Simili iniziative si andavano ad intrecciare al fenomeno cooperativo, anch'esso presente e fortemente sostenuto in Slovenia ${ }^{10}$. Successivamente, i dibattiti si svilupparono lungo tre principali linee di pensiero: una prima, ritenuta conservatrice e autoritaria, che arrivò ad elaborare una «Bozza di Legge per un'Organizzazione Corporativa Economica dello Stato», e che si rifaceva, oltre che ad una ricca letteratura internazionale, alla Carta del Lavoro italiana; una seconda, più vicina a un pensiero cattolico corporativo «modernista»; e una terza, animata dall'economista Ciril Žebot, che diede voce a una versione «radicale», esplicitamente filo-fascista, del corporativismo cattolico sloveno, ispirato direttamente dall'esperimento dei Fasci italiani e da teorici politici come Ugo Spirito ${ }^{11}$.

Anche il panorama dei partiti politici offriva numerosi gruppi che nei loro programmi e nelle pratiche organizzative rivelavano tratti corporativistici,

\footnotetext{
${ }^{8}$ Per un primo tentativo di sintesi sul caso jugoslavo: S. Petrungaro, Inter-war Yugoslavia Seen Through Corporatist Glasses, in A. Costa Pinto, Corporatism and Fascism cit., pp. 236-56. Cfr. Basciani, L'illusione della modernità cit., pp. 114-18, 165-70 e passim.

${ }^{9}$ Cfr. M. Falina, Narrating democracy in interwar Yugoslavia: From state creation to its collapse, «Journal of Modern European History», 17 (2019), n. 2, pp. 196-208.

10 Ž. Lažarević, National and Economic Features of Slovene Cooperatives, in T. Lorenz (ed.), Cooperatives in Ethnic Conflicts: Eastern Europe in the 19th and early 20th Century, Berliner Wissenschafts-Verlag, Berlin 2006, pp. 257-8.

${ }^{11} \mathrm{~J}$. Perovšek, Idejni, socialnogospodarski in narodnopolitični nazori slovenskega meščanstva v času med svetovnima vojnama (1918-1941), «Zgodovinski časopis», 51 (1997), n. 4, p. 550; M. Zver, Korporativizem v slovenski politični misli v 20. in 30. letih, «Casopis za kritiko znanosti», 20 (1992), 148/149, pp. 37-45.
} 
come ad esempio il Partito Puro del Diritto (Čista stranka prava), influenzato dall'ideologia del movimento cristiano-sociale di Karl Lueger in Austria, o il principale partito croato del periodo, il Partito Contadino (Seljačka stranka) guidato da Stjepan Radić ${ }^{12}$. Ma all'interno del panorama politico jugoslavo interbellico si trovano fermenti corporativi anche in ambienti con orientamenti ideologici assai differenti. Un buon esempio è quello di ORJUNA, l'Organizzazione dei Nazionalisti Jugoslavi (Organizacija Jugoslavenskih Nacionalista), che esistette tra 1921 e 1929. ORJUNA, rappresentante quindi non di movimenti nazionali anti-jugoslavi, ma al contrario di carattere jugoslavista, fu inizialmente influente in certe aree slovene, croate e della Vojvodina, diffondendosi poi in tutto il paese, soprattutto in Serbia, arrivando a contare nel 1923 circa 100.000 membri. Anche nel suo programma politico si rilevano elementi di stampo corporativo ${ }^{13}$.

$\mathrm{Nel}$ contesto serbo il ragionamento va inquadrato all'interno delle ricche tradizioni locali di cooperativismo contadino, sviluppate anche sotto l'influsso storico dei movimenti slavofili e populisti filorussi ${ }^{14}$. L'aspetto rilevante qui è che essi offrirono numerosi punti di contatto con i teorici del cooperativismo. Un primo esempio è quello dell'agrarianismo dal sapore corporativista elaborato da Milorad Nedeljković, che diede vita al Movimento Agrario ${ }^{15}$. Ideali più esplicitamente corporativisti rappresentavano poi le basi ideologiche di Dimitrije Ljotić, politico serbo che nel 1935 fondò il Movimento Nazionale Jugoslavo filofascista «Zbor», che durante la seconda guerra mondiale collaborò con le autorità tedesche nella Serbia occupata ${ }^{16}$.

A queste considerazioni possono esserne aggiunte altre riguardo un aspetto finora largamente trascurato dalla ricerca e che riguarda la presenza di elementi corporativi in certe riflessioni teoriche sulle nuove politiche economiche e sociali da adottare nella Jugoslavia monarchica. Si prenda ad esempio Slobodan Ž. Vidaković, influente sociologo e riformatore sociale, il quale si profuse per sviluppare una nuova e moderna politica sociale statale. Non solo la terminologia, che si serviva di nozioni quali corporazioni (korporacije), ma più in generale gli strumenti teorici di cui si avvaleva, rivelano la significativa influenza di una concezione organicistica della società, arrivando a postulare la

\footnotetext{
${ }^{12}$ T. Cipek, Liberalizam - korporativizam: dva lica ideologije hrvatskih političkih elita u Kraljevini Srba, Hrvata i Slovenaca, in H.-G. Fleck-I. Graovac (eds), Dijalog povjesničara-istoričara 3, Friedrich Naumann Stiftung, Zagreb 2001, pp. 275-90.

${ }_{13} \mathrm{~S}$. Đurašković, Ideologija Organizacije jugoslovenskih nacionalista (Orjuna), «Časopis za suvremenu povijest», 2011, n. 1, pp. 225-47.

${ }_{14}$ M. Vučković, Istorija zadružnog pokreta u Jugoslaviji, 1918-1941, Institut društvenih nauka, Beograd 1966.

${ }^{15}$ A. Stojanović, Planning a Social Transformation: A Contribution to the Research of WWII Collaboration in Serbia, «Tokovi istorije», 2013, n. 1, pp. 147-8.

${ }^{16}$ J. Byford, Willing Bystanders: Dimitrije Ljotić, 'Shield Collaboration' and the Destruction of Serbian Jews, in R. Haynes, M. Rady (eds), In the Shadow of Hitler: Personalities of the Right in Central and Eastern Europe, Bloomsbury, London 2011, pp. 295-312.
} 
necessità di forme di «terapia sociale», per promuovere «una nuova idea di Stato» e una rigenerazione dell' «organismo sociale» ${ }^{17}$.

Se Vidaković si faceva interprete di un nuovo interventismo statale più o meno corporativista, altri autori erano assai più espliciti, come l'influente economista Ljubomir Kosier, direttore della Banca Generale di Credito Croata e della Banca Centrale, con sede a Zagabria. Egli pubblicò diverse monografie in più lingue europee, incluso Stato e economia: la questione della rappresentanza corporativista ${ }^{18}$. L'opera include diverse prefazioni redatte da Carlo Sforza, già ministro italiano degli Esteri; Nikolaos Politis, ex ministro degli Esteri greco, che occupò anche importanti posizioni presso la Lega delle Nazioni; lo storico, politico e scrittore rumeno Nicolae Iorga. Kosier rigettava decisamente l'idea di trasferire il modello fascista italiano direttamente ad altri paesi e, pur difendendo il parlamentarismo e certe forme di democrazia, era convinto della necessità di un deciso interventismo statale in ambito economico, arrivando a teorizzare l'istituzione di un «consiglio economico» che affiancasse il governo con poteri consultivi.

Idee come queste, che tra l'altro non erano inedite nello spazio jugoslavo (si vedano i primi tentativi di introdurre un simile organo nel Regno di Serbia nel 1891 e in quello di Montenegro nel 1906), non furono solo oggetto di dibattiti politici e delle scienze sociali. Dopo la Grande guerra furono tradotte in pratica, con la proposizione a più riprese $(1920,1921,1932,1933)$ di un consiglio economico, «organo consultivo autonomo, costituito da esperti di questioni economiche e sociali» ${ }^{19}$. Questa istituzione, estremamente debole, aveva come funzione, almeno in teoria, di conciliare i divergenti interessi dei vari gruppi socio-professionali.

Inoltre, le Camere del Lavoro, a differenza di istituzioni con denominazioni simili che altrove in Europa costituivano l'espressione del movimento operaio organizzato, qui erano una branca del Ministero per le Politiche Sociali e la Salute Pubblica, e avevano come scopo quello di monitorare sull'applicazione delle nuove leggi relative alle assicurazioni sociali e di mediare in caso di conflitti sul posto di lavoro. Le Camere del Lavoro si svilupparono con una struttura analoga a quella dei sindacati e in una certa concorrenza con essi, con la radicale differenza che esse erano appunto un organo statale ${ }^{20}$.

Anche i datori di lavoro e i liberi professionisti potevano contare su propri organi pubblici di rappresentanza collettiva, al cui vertice fu posto l'Istituto centrale delle corporazioni industriali del Regno di Jugoslavia (Centrala industrijskih korporacija Kraljevine Jugoslavije), con sede a Belgrado $(1923)^{21}$. Istituito dal Ministero del Commercio e dell'Industria, avevano la

${ }^{17}$ S. J. Vidaković, Naši socijalni problemi, G. Kon, Beograd 1932, pp. 7-19.

$18 \mathrm{Lj}$. Kosier, Staat und Wirtschaft: Das Problem der berufsständischen Repräsentation, Bankarstvo, Berlin-Zürich 1935, $3^{\text {a }}$ ed. (prima ed. 1934).

${ }_{19}^{19}$ Ivi, pp. 95-96.

20 M. Milenković, Radničko zakonodavstvo i radničke socijalne institucije u Jugoslaviji 1918-1941, «Tokovi istorije», 1999, n. 1-4, pp. 106-17.

${ }^{21}$ Ivi, pp. 147-48. 
funzione di mediare tra i suoi membri, ossia i rappresentanti delle associazioni locali di industriali, commercianti e artigiani, e le autorità centrali dello Stato.

Quelli richiamati sono solo esempi di strutture istituzionali della Jugoslavia interbellica ancora da studiare adeguatamente, ma che forniscono alcuni importanti spunti di riflessione anche per la storia post 1945 , in cui si avrà la creazione - non proprio ex nihilo - di regimi politici fortemente caratterizzati da principi corporativi, sia nella Croazia ustascia, che nella Serbia del generale Nedić e di Ljotić ${ }^{22}$.

Riassumendo, il quadro complessivo che emerge non permette certo di sostenere che la Jugoslavia interbellica sia stata uno stato propriamente corporativo. Tuttavia, un alto numero di attori sociali e politici, provenienti da tradizioni culturali profondamente differenti, discutevano di possibili riforme corporative dello Stato jugoslavo. Non solo discorsi e riflessioni teoriche dimostrano di essere state ispirate da principi corporativisti, ma anche un certo numero di movimenti politici, strutture di partito, e persino istituzioni governative.

\section{Oltre l'opposizione stato-società civile}

Un altro ambito che ci permette di esplorare la porosità delle relazioni fra attori pubblici e privati, e la complessità delle forme di governance della prima Jugoslavia, è quello dell'associazionismo ${ }^{23}$. Esistono ormai numerosi studi su singole organizzazioni, quali - per fare solo alcuni esempi - le reti associative femminili e femministe, le associazioni culturali più importanti delle principali città, le associazioni musulmane bosniache, le società ginniche Sokol, o i veterani di guerra. Un'attenzione particolare è stata dedicata alle associazioni che rappresentavano interessi specifici come quelli delle minoranze nazionali tedesche e ungheresi, degli indipendentisti macedoni, o dei gruppi albanesi. Tuttavia, le opere di sintesi sulla prima Jugoslavia, sebbene alcune vadano ben oltre i limiti di una mera storia politica ${ }^{24}$, tendono a non dedicare grande attenzione agli attori non-governativi. Inoltre, se si escludono alcune notevoli eccezioni, gli studi che si occupano specificamente di associazioni lo fanno spesso in una prospettiva marcatamente monografica e settoriale, dedicando

${ }^{22}$ H. Sundhaussen, Wirtschaftsgeschichte Kroatiens im nationalsozialistischen Großraum 1941-1945: das Scheitern einer Ausbeutungsstrategie, Deutsche VerlagsAnstalt, Stuttgart 1983, pp. 239-43; M. Ristović, General M. Nedić. Diktatur, Kollaboration und die patriarchalische Gesellschaft Serbiens 1941-1944, in E. Oberländer (Hrsg.), Autoritäre Regime in Ostmittel- und Südosteuropa 1919-1944, Schöningh Verlag, Padeborn 2001, pp. 646-50.

${ }^{23}$ Una prima messa a punto: F. Giomi-S. Petrungaro (eds.), Voluntary associations in Yugoslavia, 1918-1941, «European Review of History/Revue européenne d'histoire», Special Issue, 26 (2019), 1. Rimandiamo a questo fascicolo per riferimenti bibliografici più puntuali.

${ }^{24}$ H. Sundhaussen, Geschichte Jugoslawiens 1918-1980, Kohlhammer, Stuttgart 1982; J. R. Lampe, Yugoslavia as History. Twice There Was a Country, Cambridge UP, Cambridge 1996; M.J. Calic, Geschichte Jugoslawiens im 20. Jahrhundert, Beck, Munich 2010. 
cioè volumi a singole associazioni, reti associative, o a tipologie di associazioni (nazionaliste, femministe, ecc.), senza indagare attentamente in che misura queste realtà fossero gli snodi di fenomeni più ampi e complessi ${ }^{25}$. Allo stesso tempo, studi come questi hanno il merito incontestabile di mostrare quanto lo spazio jugoslavo abbia partecipato allo sviluppo, in particolare a partire dalla metà del XIX secolo e fino a metà del XX secolo, della storia globale del lavoro volontario. Essi mostrano come le associazioni abbiano innervato le società urbane della regione ben prima del 1918, quando l'Impero asburgico e quello ottomano si spartivano, insieme al Regno serbo e montenegrino, la sovranità sulla regione, e quanto questa cultura associativa abbia saputo sopravvivere e adattarsi alle guerre e ai cambiamenti delle frontiere politiche.

Oltre a presentare materiali empirici molto interessanti, questi studi hanno anche un altro merito: quello di mostrare come istituzioni statali e associazioni abbiano sempre avuto delle relazioni straordinariamente complesse e mutevoli, e quanto sia fuorviante concepirle come due realtà ermeticamente separate. In altre parole, questi testi invitano lo studioso a pensare al di là della nozione di «società civile». In effetti, stando alla letteratura prodotta da scienziati politici e storici che hanno lavorato su questi temi, le associazioni sono di solito pensate come gruppi istituzionalizzati di individui, caratterizzati da due tratti principali: di essere autonomi dal potere statale, e di non avere come finalità primaria il profitto. L'obiettivo di questa definizione è quello di distinguere le associazioni, da un lato, dalle organizzazioni governative, che hanno come proprio obbiettivo quello di implementare le politiche decise nelle istituzioni pubbliche; dall'altro, dalle imprese con scopi di $1 \mathrm{ucro}^{26}$. In questa lettura ormai classica, le associazioni sarebbero da considerare come gli attori per eccellenza della cosiddetta società civile, definita come spazio per l'azione individuale e collettiva sottratta al controllo dello stato ${ }^{27}$.

Trattandosi di Europa sud-orientale e post-comunista, è opportuno tenere anche presente una peculiare valenza sociale e politica acquisita dal dibattito pubblico relativo alla storia della «società civile» in quest'area, e che ha avuto anche evidenti ricadute in termini storiografici. In Jugoslavia, come altrove in Europa orientale, il collasso dei regimI socialisti dopo il 1989 ha infatti reso la ricerca sulla società civile un tema particolarmente attraente, soprattutto per il

${ }^{25} \mathrm{M}$. Agulhon, L'histoire sociale et les associations, «Revue de l'économie sociale», 14 (1998), pp. 35-44.; G. Morton-B. de Vries-R.J. Morris (eds.), Civil Society, Associations and Urban Places: Class, Nation and Culture in Nineteenth-Century Europe, Routledge, Aldershot 2006, pp. 1-16.

${ }^{26}$ P. Clark, British Clubs and Societies, 1580-1800: The Origins of Associational World, Oxford UP, New York 2000, p. 16; J. Bradley, Voluntary Associations in Tsarist Russia. Science, Patriotism and Civil Society, Cambridge UP, Cambridge 2009, pp. 56.

${ }^{27}$ J. Kocka, Zivilgesellschaft als historisches Problem und Versprechen, in M. Hildermeier-J. Kocka-C. Christoph Conrad (Hrsg.), Europäische Zivilgesellschaft in Ost und West. Begriff, Geschichte, Chancen, Campus Verlag, Frankfurt a.M. 2000, pp. 13-39; A. Bauerkämper (Hrsg.) Die Praxis der Zivilgesellschaft. Akteure, Handeln und Strukturen im internationalen Vergleich, Campus Verlag, Frankfurt a.M. 2003. 
periodo socialista, e ancor più per quello post-socialista ${ }^{28}$. A partire almeno dai tardi anni Settanta, questa nozione fu infatti altamente politicizzata e legata ai movimenti d'opposizione anticomunista come Solidarnošć in Polonia, Charta 77 in Cecoslovacchia, e certi gruppi intellettuali nell'Ungheria tardo-socialista. Sia nei dibattiti accademici che nel discorso comune, l'idea di società civile fu connessa alle speranze di una rigenerazione sociale e politica, spesso con un'idea normativa di democrazia occidentale e dell'economia neoliberale, che si sarebbero dovute introdurre rapidamente negli stati post-comunisti. La questione della relazione tra associazioni volontarie e lo stato acquista così, in queste regioni d'Europa, implicazioni teoriche e interpretative aggiuntive, soprattutto se declinata in un contesto storico di più lungo respiro, che raggiunga anche l'epoca precedente all'avvento del socialismo. In che misura la società civile di questi paesi fu repressa dai partiti comunisti, rinascendo dopo l'Ottantanove? Vi era una società civile prima del secondo conflitto mondiale? Se sì, quali ne erano le caratteristiche? ${ }^{29}$

Già durante gli anni '20, quando la Jugoslavia è una monarchia parlamentare che comunque riserva al re un ampio spazio di manovra nella vita politica, Stato e associazioni volontarie entrano in relazioni molteplici e complesse, che rendono di fatto impossibile tracciare una linea di demarcazione netta fra i due termini. Il mondo associativo conosce profonde trasformazioni: alcune reti associative fondate prima della Grande Guerra - associazioni nazionaliste, filantropiche, femministe - conoscono processi di convergenze, fusioni e spaccature lungo linee politiche nuove.

Subito dopo la Grande Guerra, le autorità del nuovo stato mobilitano nei confronti del nuovo stato un discorso tipicamente liberale: il lavoro volontario dei cittadini è da considerarsi come una risorsa importante per il progresso e l'unificazione dei differenti segmenti della società jugoslava in formazione. Nella pratica, però, le relazioni dello stato e delle sue articolazioni locali con questo panorama associativo in riconfigurazione sembrano essere straordinariamente diversificate. In certi casi, associazioni percepite come vicine ai partiti di opposizione, o come infedeli al progetto di costruzione di uno stato unitario per serbi, croati e sloveni, vengono regolarmente ostacolate, o comunque tenute sotto controllo dalla polizia o dall'esercito. È il caso delle associazioni delle minoranze nazionali e religiose, ma anche di organizzazioni vicine a partiti d'opposizione, come il Partito contadino croato o l'Organizzazione Musulmana Jugoslava. In alcune occasioni, come nel caso dell'assemblea di ripresa dei lavori dopo la guerra della principale associazione

${ }^{28}$ K. Jacobsson, Urban Movements and Grassroots Activism in Central and Eastern Europe, Ashgate, Farnham 2015; S. Fischer, H. Pleines (eds.), Civil society in Central and Eastern Europe, Ibidem Verlag, Stuttgart 2014; J. Hackmann-K. Roth (Hrsg.), Zivilgesellschaft im östlichen und südostlichen Europa in Geschichte und Gegenwart, Oldenbourg, München 2011.

${ }^{29}$ W. Höpken, Stadt und Zivilgesellschaft in Südosteuropa: Anmerkungen aus historischer Perspektive, in A. Sterbling (Hrsg.), Zivilgesellschaftliche Entwicklungen in Südosteuropa, Otto Sagner, München 2009 pp. 111-56. 
culturale musulmana del paese, Gajret (Lo Zelo) del 1919, la polizia di Sarajevo interviene per permettere ad elementi vicini ai partiti di governo di assicurarsi il controllo dell'associazione, nonostante le proteste dei partecipanti. In altri casi, come ad esempio la Federazione delle associazioni giovanili ebraiche jugoslave (Savez židovskih omladinskih udruženja), le interazioni fra stato e associazione sembrano esser state straordinariamente limitate, improntate ad una indifferenza reciproca. In altri casi ancora, governo e corona sostengono direttamente diverse associazioni, non solo quelle di tipo filantropico, ma anche culturali, riconoscendo il loro ruolo ausiliario in ambiti nei quali il nuovo stato ancora faticava a porre in essere politiche efficienti, come l'educazione e la protezione sociale. Il legame fra Belgrado e queste associazioni disseminate sul territorio prende la forma di vero e proprio sostegno finanziario, logistico e simbolico, talvolta formalizzato attraverso l'istituto del «patronato» (pokroviteljstvo) da parte dei membri della famiglia reale $^{30}$.

Questa vasta panoplia di interazioni stato-associazioni, che poteva andare dal controllo, all'opposizione, all'indifferenza e alla collaborazione, conosce una trasformazione radicale con l'inizio della dittatura reale. Si tratta di una svolta che, oltre ad abolire la costituzione e sciogliere il parlamento, assegnando tutto il potere al re e al governo da lui dominato, ridisegna la carta amministrativa del paese, mettendo fuori legge qualsiasi organizzazione a carattere etno-confessionale e non jugoslavista. La società jugoslava viene sottoposta a un controllo poliziesco molto più elevato di quello conosciuto nei dieci anni precedenti. In questo nuovo scenario, le associazioni - o meglio, alcune di esse - sembrano aver giocato un ruolo di primo piano. Fra di esse vanno senza dubbio ricordate i Sokoli, alle quali si impone in questo momento una organizzazione unitaria e con una agenda apertamente jugoslavista, ma anche altre associazioni come Jadranska straža, pensata per promuovere l'orientamento marittimo e adriatico del paese. Anche altre associazioni entrano in una relazione privilegiata con lo stato post-liberale: Gajret, la già ricordata associazione culturale musulmana, come pure Kolo Srpskih Sestara, associazione filantropica femminile serbo-ortodossa, oppure Zveza slovenskih vojakov, organizzazione dei veterani particolarmente forte radicata in Slovenia, o ancora le associazioni dei vigili del fuoco volontari ${ }^{31}$. Pur nella loro grande diversità, queste associazioni sembrano ricoprire un ruolo simile: in un mondo dove il parlamento è chiuso o sotto controllo, esse entrano in una nuova relazione con lo stato e con esso siglano un nuovo patto. Lo stato accorda a queste associazioni finanziamento, relazioni privilegiate con i media e le amministrazioni territoriali, privilegi materiali e simbolici; in cambio, gli uomini e le donne che investono tempo ed energie in queste reti associative si

\footnotetext{
${ }^{30}$ Cfr. i contributi di Bojan Mitrović, Bernd Robionek e Stefano Petrungaro in GiomiPetrungaro, Voluntary associations in Yugoslavia cit.

${ }^{31}$ Si rinvia ai contributi di Igor Tchoukarine, Ana Kladnik e Fabio Giomi in ivi, e J. P. Newman, Yugoslavia in the Shadow of War: Veterans and the Limits of State Building, 1903-1945, Cambridge U.P., Cambridge 2015.
} 
fanno propagatori - pur con gradi diversi di sincerità e dedizione dell'ideologia jugoslavista, ottenendo in cambio riconoscimenti da parte dell'amministrazione. Si verifica insomma una governamentalizzazione di queste associazioni, i cui leader vanno talvolta a ricoprire ruoli di primo piano nelle amministrazioni provinciali o finanche nel governo. Una volta riconosciuti come nacionalni radnici (lett. «lavoratori nazionali», che operavano cioè negli interessi della Nazione), le carriere d'attivista e quelle amministrative si sovrapponevano.

Anche se alcuni di questi tratti cambiano dopo l'assassinio del re nel 1934, al quale segue un rilassamento della dittatura, ci sembra che queste dinamiche permangano fino alla fine della prima Jugoslavia: le associazioni continuano a venir cooptate dallo stato, diventando un pilastro, un corpo intermedio alternativo a quelli - considerati come infidi o superati - dei partiti politici e del parlamento. La frontiera fra Stato e società civile diventa così del tutto sfumata.

\section{Verso una nuova alleanza trono-altare}

La relazione fra Stato e istituzioni religiose sembra aver seguito una traiettoria simile. Le autorità religiose che dovevano occuparsi del variegato panorama religioso jugoslavo - composto, ricordiamolo, da una maggioranza relativa di cristiano-ortodossi, seguiti da cristiano-cattolici, musulmani, ebrei e protestanti, solo per limitarsi ai gruppi principali - conobbero una relazione mutevole con il nuovo stato, per certi versi paragonabile a quella delle associazioni volontarie. A costo di una certa semplificazione, ci pare di poter affermare che la ridefinizione della relazione fra stato e autorità religiose passa, nei decenni interbellici, attraversò tre snodi principali.

Un primo momento da prendere in conto è quello che seguì immediatamente la Grande guerra, nel quale le diverse forze politiche discussero e la prima Costituzione del paese nel giugno del 1921. Nei mesi che precedettero l'approvazione, durante le discussioni dell'Assemblea Costituente i diversi attori nazionali (leader politici e religiosi, corona) e internazionali (il Vaticano in particolare) discussero i principi sui quali fondare la relazioni fra il nuovo stato jugoslavo e le autorità religiose già presenti nel paese. La diversità dei punti di vista non tardò a manifestarsi tanto sulla stampa del paese che in seno all'Assemblea: il patriarca di Belgrado, per esempio, non aveva esitato a chiedere che la nuova Costituzione riconoscesse alla Chiesa ortodossa lo status di chiesa dominante previsto dalla costituzione serba del 1903, mostrando di fatto di concepire il nuovo regno come una semplice espansione territoriale del Regno di Serbia, e non come l'espressione di un nuovo patto politico; la Chiesa Cattolica, per bocca del nunzio apostolico e dei vescovi, si era espressa contro questa prospettiva, chiedendo che le diverse istituzioni religiose fossero tutte uguali di fronte allo stato, e allo stesso tempo che la costituzione sancisse esplicitamente il diritto dei vescovi jugoslavi ad avere relazioni pienamente 
libere con Roma. ${ }^{32}$ Entrambe le richieste andarono deluse: 1'articolo 12 della Costituzione, in effetti, sancì il principio della libertà di religione per tutti i culti riconosciuti (priznate vjeroispovjesti) su una base di uguaglianza (ravnopravnost), senza menzionare il diritto del clero cattolico di avere libere relazioni con Roma. Dal 1921 la prima Jugoslavia si presentò quindi come uno stato «a-confessionale», impegnato a riconoscere l'autonomia organizzativa delle istituzioni religiose «nei limiti della legge», e a sostenere economicamente le istituzioni religiose «sulla base del numero dei fedeli e delle necessità». Come afferma Pieter Troch, «a differenza del tipico modello est europeo di una chiesa-una nazione, tutte le religioni [riconosciute] erano concepite come chiese nazionali parallele, che dovevano essere sottoposte al controllo dello stato» ${ }^{33}$.

Nei mesi successivi, il Parlamento avrebbe dovuto tradurre in apposite leggi e regolamenti queste disposizioni generali, in modo da far tabula rasa del mosaico legislativo ereditato dal passato. In effetti, fino a quel momento le relazioni fra stato e istituzioni religiose continuavano ad essere gestite attraverso un mosaico di leggi, regolamenti, accordi e concordati adottati prima del 1914 e che creavano enormi disparità e incoerenze fra culti e regioni. Più facile a dirsi che a farsi: le tensioni fra partiti politici, ai quali si aggiungevano le visioni inconciliabili delle varie istituzioni del paese, avrebbero reso impossibile l'adozione di queste leggi per tutti gli anni '20. Il risultato fu quindi paradossale: uno stato a-confessionale, dei culti riconosciuti, e le loro relazioni gestite da un patchwork di norme concepiti in contesti politici precedenti ${ }^{34}$.

Il quadro cambiò radicalmente con l'inizio della dittatura reale. I mesi a cavallo fra 1929 e 1930, lo stato - più precisamene il Ministero della Giustizia, che aveva da poco assorbito le funzioni del Ministero dei Culti - riconfigurò la relazione con le istituzioni religiose. Seguì la promulgazione, da parte del Governo, di una serie di leggi che regolavano le relazioni con lo stato e l'organizzazione interna della maggior parte delle autorità religiose presenti nel paese: la chiesa ortodossa (8 dicembre 1929), la comunità ebraica (14 dicembre 1929), la comunità islamica (31 gennaio 1930), le chiese evangelica e riformata (14 aprile 1930). Come affermato dallo storico del diritto Mustafa Imamović, che ha analizzato questo corpus legislativo nel dettaglio, al di là delle differenze queste leggi sembrano rispondere ad una logica comune: riscrivere il patto fra stato e autorità religiose a vantaggio netto (ma non esclusivo) del primo sulle seconde $^{35}$. Le organizzazioni religiose riconosciute scivolarono di fatto sotto il

\footnotetext{
${ }^{32}$ M. Imamović, Pokušaj donošenja interkonfesionalnog zakona u Kraljevini Srba, Hrvata i Slovenaca, «Glasnik Rijaseta Islamske zajednice u RBiH», 1994, nn. 4-6, pp. 235-49.

${ }^{33}$ P. Troch, Nationalism and Yugoslavia. Education, Yugoslavism and the Balkans before World War II, I.B. Tauris, London 2015, p. 114.

34 E. Greble, The Uncertain "Wilsonian Moment" for Muslims in Yugoslavia: Reframing Historiographical Conversations through Minority Experiences, «Passato e presente», 2019, pp. 27-36.

${ }_{35}$ M. Imamović, Vjerske zajednice u Bosni i Herzegovini u Jugoslaviji između dva svjetska rata, Pravni fakultet Univerziteta u Sarajevu, Sarajevo 2008.
} 
controllo del Ministero della Giustizia, che si riservò quasi sempre un diritto di nomina dei vertici, o comunque un ampio diritto di interferenza. Nel caso delle istituzioni musulmane, il governo arrivò addirittura nel 1930 a spingere al pensionamento il reis-ul-ulema in carica, Mehmed Džemaludin Čaušević, e a nominare al suo posto un leader apertamente filo-governativo, Ibrahim Maglajlić $^{36}$. Tuttavia, sarebbe un errore pensare che le istituzioni religiose ne trassero esclusivamente svantaggi: come contropartita esse ottennero l'abolizione del matrimonio civile, in vigore fino ad allora in alcune regioni post-asburgiche, e quindi il monopolio in materia matrimoniale; i registri delle istituzioni religiose diventarono documenti di diritto pubblico; ma soprattutto, tutti i progetti di marginalizzazione o addirittura eliminazione della religione e dei religiosi dall'insegnamento scolastico statale, che erano invece stati regolarmente discussi negli anni '20, suscitando un'opposizione trasversale delle istituzioni religiose, vennero definitivamente abbandonati. Le principali ragioni sottese a questo cambiamento nelle relazioni fra stato e istituzioni religiose furono da un lato, la paura del separatismo nazionale e del ruolo che le istituzioni religiose potevano giocare, e spesso giocavano, in questa polarizzazione; dall'altro, il timore (che assumeva a tratti i colori della paranoia) del comunismo e della sua diffusione presso le nuove generazioni, in particolare quelle dotate di una educazione media o superiore. Più che una semplice sottomissione delle istituzioni religiose allo stato, siamo di fronte alla scrittura di un nuovo patto: le istituzioni religiose divennero istituzioni paragovernative, ulteriori corpi intermedi fra il re e i differenti gruppi religiosi nei quali i suoi sudditi erano suddivisi.

Questo nuovo patto fra stato e istituzioni religiose, tuttavia, non funzionò sempre e non funzionò per tutti. Se prendiamo il caso della Comunità islamica, vediamo che la situazione descritta sopra perdurò solo fino al 1936, quando la morte de re e l'inizio di una nuova stagione di parlamentarismo limitato imposero ai governi di Belgrado di scendere a patti con il principale attore politico dei musulmani di Bosnia, Mehmed Spaho, e di cooptarlo nel governo. In cambio, la corona accettò che le attività della Comunità islamica jugoslava fossero organizzate da un nuovo statuto che mise di fatto le istituzioni religiose sotto il controllo degli uomini vicini all'Organizzazione Musulmana Jugoslava, il partito di Spaho. L'elezione alla carica di reis-ul-ulema due anni dopo di Fehim Spaho, fratello di Mehmed, suggellò la sottomissione della gerarchia religiosa islamica del paese alla politica. Le istituzioni religiose islamiche passarono quindi da essere para-statali a para-partitiche ${ }^{37}$.

I limiti di questo nuovo corso nelle relazioni fra stato e istituzioni religiose diventa ancora più tangibile nel caso delle relazioni con la Chiesa cattolica, autentica spina nel fianco della politica religiosa jugoslava interbellica. Il problema principale in questo caso era rappresentato dal fatto che il vertice di

${ }^{36}$ A. Jahić, Islamska zajednica u Bosni i Hercegovini za vrijeme monarhističke republike Jugoslavije (1918-1941), Islamska Zajednica u Hrvatskoj e Medžlis Islamske Zajednice u Zagrebu, Zagreb 2010.

${ }^{37}$ A. Jahić, Islamska zajednica cit., pp. 535-65. 
questa organizzazione religiosa non fosse all'interno della Jugoslavia, e quindi controllabile, e - dopo il 1929 - vicina all'Italia di Mussolini, grande avversario regionale della Jugoslavia. In questo caso, tutti i tentativi di arrivare ad un patto ad hoc con il clero cattolico jugoslavo fallirono: discusso a partire dalla metà degli anni ' 20 , un progetto di concordato - che pure era stato firmato dalle due parti nel 1935 - non sarà mai ratificato dal parlamento, in seguito all'opposizione durissima dei partiti politici serbi e - soprattutto - della Chiesa ortodossa ${ }^{38}$.

\section{La Jugoslavia nell'Europa post-liberale: un'agenda di ricerca}

Queste riflessioni, per quanto ancora ad uno stato embrionale, vogliono essere un invito ad analizzare la Jugoslavia degli anni ' 20 e ' 30 come un periodo storico molto più complesso di quello che si scrive di solito, ossia un lento $\mathrm{e}$ inesorabile scivolamento verso l'implosione statuale e il conflitto nazionale e civile. Pur non producendo uno dei tanti «-ismi» che affollano la storia delle idee del periodo interbellico - fascismo, nazismo, stalinismo, kemalismo ecc. - la Jugoslavia presenta forme di governance di grande interesse, che portano ad una costante e complessa rielaborazione delle relazioni fra stato e società. Letti nell'ottica qui proposta, persino gli anni ' 30 , quelli cioè maggiormente legati a pratiche autoritarie, appaiono molto più che un momento meramente repressivo alla periferia d'Europa. Quegli anni, infatti, sono testimoni di una ricerca avviata già nel decennio precedente e volta a individuare, istituzionalizzare e testare forme originali di cogestione della collettività politica, economica e culturale. Enti governativi, associazioni private, istituzioni religiose, strutture partitiche e organizzazioni professionali, iniziano a interagire in forme in parte inedite e senz'altro intense, che rivelano come il confine tra privato e pubblico, sfera governativa e società civile, fosse in continua rinegoziazione. Il caso jugoslavo, e con tutta probabilità l'intera regione sud-est-europea, sembra quindi prezioso per esplorare in chiave comparata e transnazionale il panorama delle risposte europee alla crisi del liberalismo tra le due guerre mondiali.

\footnotetext{
${ }^{38}$ M. Imamović, Vjerske zajednice cit., pp. 39-51; K. Buchenau, Kämpfende Kirchen. Jugoslawiens religiöse Hypothek, Peter Lang, Frankfurt am Main 2006; M. Falina, Svetosavlje: A Case Study in the Nationalization of Religion, «Schweizerische Zeitschrift für Religions- und Kulturgeschichte», 101 (2007), pp. 505-27.
} 